\title{
Neutron stars in scalar-tensor theories of gravity and catastrophe theory
}

\author{
Tomohiro Harada 周 \\ Department of Physics, Kyoto University, Kyoto 606-01, Japan
}

\begin{abstract}
We investigate neutron stars in scalar-tensor theories. We examine their secular stability against spherically symmetric perturbations by use of a turning point method. For some choices of the coupling function contained in the theories, the number of the stable equilibrium solutions changes and the realized equilibrium solution may change discontinuously as the asymptotic value of the scalar field or total baryon number is changed continuously. The behavior of the stable equilibrium solutions is explained by fold and cusp catastrophes. Whether or not the cusp catastrophe appears depends on the choice of the coupling function. These types of catastrophes are structurally stable. Recently discovered spontaneous scalarization, which is a nonperturbative strong-field phenomenon due to the presence of the gravitational scalar field, is well described in terms of the cusp catastrophe.
\end{abstract}

PACS numbers: 04.40.Dg, 04.50.+h, 05.70.Fh, 97.60.Jd

\section{INTRODUCTION}

Scalar-tensor theories [1.2] are among the generalized theories of gravitation. Brans-Dicke theory [3] is a member of the scalar-tensor theories. Scalar-tensor theories have recently attracted the attention of many researchers. One of the reasons is that the unified theories that contain gravity as well as other interactions, such as string theory [ 1 , naturally predict the existence of scalar fields that relate to gravity. In the hyperextended inflation model [5], scalar-tensor theories of gravity play an essential role. Moreover, projects of laser interferometric gravitational wave observations [6. 9] will be soon in practical use, so that high-accuracy tests of the scalar-tensor theories may be expected 10 14.

Scalar-tensor theories are viable theories of gravity for some choices of the coupling function which is contained in the theories. Predictions of these theories in a strong field may be drastically different from those of general relativity. Recently, Damour and Esposito-Farèse [15,16] discovered one example of such phenomena. They showed that, for some choices of the coupling function, the configuration of a massive neutron star deviates significantly from that in general relativity, even if the post-Newtonian limit of the theory is extremely close to or even agrees with that of general relativity. This deviation in a strong field may be easily tested from binary-pulsar timing observations, if it exists, because of the extra energy loss by scalar gravitational radiation [16]. The deviation from general relativity can be no longer dealt with as a perturbative effect from general relativity. Damour and Esposito-Farèse referred to this nonperturbative strong-field effect as "spontaneous scalarization" in analogy to the spontaneous magnetization of the ferromagnets.

In this paper, we investigate spontaneous scalarization in detail with the technique of catastrophe theory. A many-parameter version of the turning point method 17 20 is used as a tool of a stability analysis of equilibrium solutions. The stability analysis of boson stars in scalar-tensor gravity via catastrophe theory in the case of onedimensional control space was done in [21,22]. Catastrophe types of neutron star equilibrium solutions are classified as fold and cusp catastrophes. The occurrence of the cusp catastrophe depends on the choice of the coupling function. Spontaneous scalarization is classified as the cusp catastrophe. From this catastrophic feature, we conclude that the stable configuration of the neutron star may change discontinuously as the baryon number of the star or the asymptotic value of the scalar field changes continuously. The behavior of the scalar charge around the cusp point is

\footnotetext{
* Email address: harada@tap.scphys.kyoto-u.ac.jp
} 
explained by catastrophe theory. For the coupling function considered here, when the asymptotic value of the scalar field is such that the theory agrees with general relativity in the post-Newtonian limit, we find the sequence of the equilibrium solutions bifurcates to three branches at some critical central density. One branch consists of solutions that are identical to neutron stars in general relativity, and the other two consist of solutions that deviate significantly from neutron stars in general relativity. The general relativistic branch is secularly unstable in agreement with the result obtained by a perturbation study [23], while the non-general-relativistic branches are secularly stable.

This paper is organized as follows. In Sec. II, we summarize the field equations of scalar-tensor theory and the equations determining equilibrium solutions of neutron stars in this gravitational theory. In Sec. III, we present stability criteria on the grounds of the turning point method. In Sec. IV, the stability criteria are applied to equilibrium solutions of neutron stars in scalar-tensor theory and some consequences of catastrophe theory are discussed. Section $\mathrm{V}$ is devoted to conclusions. We use units in which $c=1$. The Greek indices run from 0 to 3 . We follow the Misner-Thorne-Wheeler [24] sign conventions for curvature quantities.

\section{BASIC EQUATIONS}

Here we consider a class of scalar-tensor theories in which gravity is mediated by not only a metric tensor but also a massless scalar field. The action is given by [2]

$$
I=\frac{1}{16 \pi G_{*}} \int \sqrt{-g_{*}}\left(R_{*}-2 g_{*}^{\mu \nu} \varphi_{, \mu} \varphi_{, \nu}\right) d^{4} x+I_{m}\left[\Psi_{m}, A^{2}(\varphi) g_{* \mu \nu}\right]
$$

where $g_{* \mu \nu}$ is the "Einstein" frame metric tensor, $\Psi_{m}$ denotes matter fields collectively, and $G_{*}$ is some dimensionful constant. In this Einstein frame, the Einstein-Hilbert term is isolated from other sectors. The "Brans-Dicke" frame metric tensor $\tilde{g}_{\mu \nu}$ is related to the Einstein frame metric tensor by the following conformal transformation:

$$
\tilde{g}_{\mu \nu}=A^{2}(\varphi) g_{* \mu \nu}
$$

Because of the "universal coupling," which is the way of the coupling of the scalar field in the matter sector seen in Eq. (2.1), a test particle moves on the geodesic of the Brans-Dicke frame metric $\tilde{g}_{\mu \nu}$. For this reason the Brans-Dicke frame is often called a "physical" frame. The tilde denotes the physical frame quantity.

In the Einstein frame, the field equations are given by

$$
\begin{aligned}
& G_{* \mu \nu}=8 \pi G_{*} T_{* \mu \nu}+2\left(\varphi_{, \mu} \varphi_{, \nu}-\frac{1}{2} g_{* \mu \nu} g_{*}^{\alpha \beta} \varphi_{, \alpha} \varphi_{, \beta}\right), \\
& \square_{*} \varphi=-4 \pi G_{*} \alpha(\varphi) T_{*},
\end{aligned}
$$

while the equations of motion for matter are

$$
\nabla_{* \nu} T_{* \mu}^{\nu}=\alpha(\varphi) T_{*} \nabla_{* \mu} \varphi
$$

where the energy-momentum tensor of the matter, $T_{*}^{\mu \nu}$, is defined and related to the physical energy-momentum tensor $\tilde{T}^{\mu \nu}$ as

$$
T_{*}^{\mu \nu} \equiv \frac{2}{\sqrt{-g_{*}}} \frac{\delta I_{m}\left[\Psi_{m}, A^{2}(\varphi) g_{* \mu \nu}\right]}{\delta g_{* \mu \nu}}=A^{6}(\varphi) \tilde{T}^{\mu \nu} .
$$

$G_{* \mu \nu}$ and $\square_{*}$ are the Einstein tensor and d'Alembertian of $g_{* \mu \nu}$, respectively. $T_{*}$ and $\alpha(\varphi)$ are defined as

$$
\begin{aligned}
T_{*} & \equiv T_{* \mu}{ }^{\mu} \equiv T_{*}^{\mu \nu} g_{* \mu \nu}, \\
\alpha(\varphi) & \equiv \frac{d \ln A(\varphi)}{d \varphi} .
\end{aligned}
$$

The parameters in the parametrized post-Newtonian framework are given by [1,2]

$$
\begin{aligned}
& 1-\gamma_{E d d}=\frac{2 \alpha_{0}^{2}}{1+\alpha_{0}^{2}}, \\
& \beta_{E d d}-1=\frac{\beta_{0} \alpha_{0}^{2}}{2\left(1+\alpha_{0}^{2}\right)^{2}}, \\
& \xi=\alpha_{1}=\alpha_{2}=\alpha_{3}=0,
\end{aligned}
$$


where $\beta_{E d d}$ and $\gamma_{E d d}$ are the so-called Eddington parameters. We have defined

$$
\begin{aligned}
\alpha_{0} & \equiv \alpha\left(\varphi_{0}\right), \\
\beta_{0} & \equiv \frac{d \alpha}{d \varphi}\left(\varphi_{0}\right),
\end{aligned}
$$

and $\varphi_{0}$ is the value of the scalar field $\varphi$ in the spatial asymptotic region. We assume that the cosmological evolution of the scalar field is sufficiently slow in comparison with the characteristic time scale of the local gravitational process of the isolated object considered here. From this assumption $\varphi_{0}$ is regarded as the cosmological value of the scalar field. On the other hand, we can identify the asymptotic value $\varphi_{0}$ to the value of the scalar field in the matching region in the matching approach to the $N$-compact-body problem (see Appendix A of [2]). Then, the solar-system experimental constraints are [25]

$$
\gamma_{E d d}=0.9996 \pm 0.0017
$$

and 26 .

$$
4 \beta_{E d d}-\gamma_{E d d}-3=-0.0007 \pm 0.0010
$$

We summarize equations for the structure of a relativistic star in scalar-tensor theory, following [15]. We restrict ourselves to the static and spherically symmetric case. The metric is given in the following form:

$$
d s_{*}^{2}=-e^{\nu(r)} d t^{2}+\left(1-\frac{2 \mu(r)}{r}\right)^{-1} d r^{2}+r^{2}\left(d \theta^{2}+\sin ^{2} \theta d \phi^{2}\right) .
$$

The matter is described as a perfect fluid: i.e.,

$$
\tilde{T}_{\mu \nu}=(\tilde{\rho}+\tilde{p}) \tilde{u}_{\mu} \tilde{u}_{\nu}+\tilde{p} \tilde{g}_{\mu \nu}
$$

Then, the following equations are obtained:

$$
\begin{aligned}
\mu^{\prime} & =4 \pi G_{*} r^{2} A^{4} \tilde{\rho}+\frac{1}{2} r(r-2 \mu) \psi^{2}, \\
\nu^{\prime} & =8 \pi G_{*} \frac{r^{2} A^{4} \tilde{p}}{r-2 \mu}+r \psi^{2}+\frac{2 \mu}{r(r-2 \mu)}, \\
\varphi^{\prime} & =\psi, \\
\psi^{\prime} & =4 \pi G_{*} \frac{r A^{4}}{r-2 \mu}[\alpha(\tilde{\rho}-3 \tilde{p})+r(\tilde{\rho}-\tilde{p}) \psi]-\frac{2(r-\mu)}{r(r-2 \mu)} \psi, \\
\tilde{p}^{\prime} & =-(\tilde{\rho}+\tilde{p})\left[4 \pi G_{*} \frac{r^{2} A^{4} \tilde{p}}{r-2 \mu}+\frac{1}{2} r \psi^{2}+\frac{\mu}{r(r-2 \mu)}+\alpha(\varphi) \psi\right], \\
\tilde{p} & =\tilde{p}(\tilde{\rho}),
\end{aligned}
$$

where the prime denotes a derivative with respect to $r$. We use the polytropic equations of state:

$$
\begin{aligned}
\tilde{\rho} & =\tilde{n} m_{b}+\frac{K n_{0} m_{b}}{\Gamma-1}\left(\frac{\tilde{n}}{n_{0}}\right)^{\Gamma}, \\
\tilde{p} & =K n_{0} m_{b}\left(\frac{\tilde{n}}{n_{0}}\right)^{\Gamma}, \\
m_{b} & =1.66 \times 10^{-24} \mathrm{~g}, \\
n_{0} & =0.1 \mathrm{fm}^{-3},
\end{aligned}
$$

where $\tilde{n}$ is the baryon number density in the Brans-Dicke frame. We then take the parameters $\Gamma=2.34$ and $K=0.0195$ (EOS II of [15]). Note that the total baryon number is given by

$$
N=\int 4 \pi \tilde{n} A^{3} r^{2}\left(1-\frac{2 \mu}{r}\right)^{-1 / 2} d r
$$


Here we present the method of solving the above equations and obtaining the structure of a neutron star. First the initial values of the above set of ordinary differential equations are fixed as

$$
\mu(0)=0, \nu(0)=0, \varphi(0)=\varphi_{c}, \psi(0)=0, \tilde{p}(0)=\tilde{p}_{c}
$$

and Eqs. (2.18)-(2.23) are integrated numerically up to the stellar surface at which $\tilde{p}=0$. Thereafter the solution is matched with the static and spherically symmetric "vacuum" solution, where the term "vacuum" means only the absence of matter, i.e., $\tilde{T}_{\mu \nu}=0$. This solution is given in [2]. The solutions are parametrized by three parameters $b$, $d$, and $\varphi_{0}$. From the matching conditions at the surface, we can obtain $\nu(r)$ including the constant term. In order to set $A\left(\varphi_{0}\right)$ to unity, we rescale the raw quantities to the renormalized ones as follows:

$$
\begin{gathered}
r_{r e n}=A_{0}^{2} r, \quad \mu_{r e n}=A_{0}^{2} \mu, \quad \nu_{r e n}=\nu, \quad \varphi_{r e n}=\varphi, \quad \psi_{r e n}=A_{0}^{-2} \psi, \\
\varphi_{\text {ren }}=\varphi_{0}, \quad a_{r e n}=A_{0}^{2} a, \quad b_{r e n}=A_{0}^{2} b, \quad d_{r e n}=A_{0}^{2} d, \quad N_{r e n}=A_{0}^{3} N .
\end{gathered}
$$

Then, from the asymptotic properties at spatial infinity for a static and isolated system

$$
\begin{aligned}
g_{* \mu \nu} & =\eta_{\mu \nu}+\frac{2 G_{*} m}{r_{r e n}} \delta_{\mu \nu}+O\left(\frac{G_{*}^{2}}{r_{r e n}^{2}}\right), \\
\varphi & =\varphi_{0}+\frac{G_{*} \omega}{r_{r e n}}+O\left(\frac{G_{*}^{2}}{r_{r e n}^{2}}\right)
\end{aligned}
$$

where $\eta_{\mu \nu}$ is the Minkowskian metric. We call $m$ the Arnowitt-Deser-Misner (ADM) energy and $\omega$ the scalar charge [2]. $b_{\text {ren }}$ and $d_{\text {ren }}$ are related to $m$ and $\omega$ as

$$
\begin{aligned}
G_{*} m & =\frac{b_{r e n}}{2}, \\
G_{*} \omega & =-d_{r e n} .
\end{aligned}
$$

Hereafter the subscripts "ren" are omitted for simplicity. We use units in which $G_{*}=1$.

\section{STABILITY CRITERIA}

In scalar-tensor theory, control parameters of the static, spherically symmetric, and isolated neutron star are not only the baryon number $N$ but also the "external field", that is, the asymptotic value of the scalar field $\varphi_{0}$. For static systems, the partial derivative of $m$ in terms of $\varphi_{0}$ with $N$ constant is given by [2]

$$
\left(\frac{\partial m}{\partial \varphi_{0}}\right)_{N}=-\omega
$$

The energy injection of the system by increasing baryons is described as

$$
\int 4 \pi \tilde{u}_{0} \delta \tilde{\rho} A^{3} r^{2}\left(1-\frac{2 \mu}{r}\right)^{-1 / 2} d r=\int 4 \pi e^{\nu / 2} \tilde{\mu} \delta \tilde{n} A^{4} r^{2}\left(1-\frac{2 \mu}{r}\right)^{-1 / 2} d r
$$

where $\tilde{\mu} \equiv d \tilde{\rho} / d \tilde{n}$ is the chemical potential. The first law of thermodynamics in an adiabatic process is

$$
d\left(\frac{\tilde{\rho}}{\tilde{n}}\right)=-\tilde{p} d\left(\frac{1}{\tilde{n}}\right)
$$

From Eqs. (2.19), 2.20), (2.22), and (3.3), we find that the quantity $A e^{\nu / 2} \tilde{\mu}$ is constant all over the star. Therefore this quantity can be estimated by its value at the stellar surface. Using this fact, the expression of the energy injection, Eq. (3.2), is rewritten as

$$
A_{s} e^{\nu_{s} / 2} \tilde{\mu}_{s} \int 4 \pi A^{3} r^{2}\left(1-\frac{2 \mu}{r}\right)^{-1 / 2} \delta \tilde{n} d r=A_{s} e^{\nu_{s} / 2} \tilde{\mu}_{s} \delta N
$$

where the suffix " $s$ " indicates that the quantity is evaluated at the stellar surface $r=r_{s}$. Therefore, the effective chemical potential, $\mu_{e f f}$, is given by 


$$
\mu_{e f f} \equiv\left(\frac{\partial m}{\partial N}\right)_{\varphi_{0}}=A_{s} e^{\nu_{s} / 2} \tilde{\mu}_{s} .
$$

From the above discussions the variation of $m$ for static systems in a quasistatic process is written in the following form:

$$
\delta m=-\omega \delta \varphi_{0}+\mu_{e f f} \delta N
$$

where by "quasistatic process" we mean successive changes among the infinitesimally nearby equilibrium solutions.

Suppose that the isolated neutron star is perturbed slightly with $\varphi_{0}$ and $N$ constant for some reason other than incident waves, while spherical symmetry is preserved. Then the outgoing waves can carry out some positive energy to infinity and the system cannot keep its original state if there exists an energetically favorable configuration which is infinitesimally deformed from the system with the same $\varphi_{0}$ and $N$. Therefore, an equilibrium solution $X$ is secularly stable against spherically symmetric (infinitesimal) perturbations if and only if there is no momentarily static and spherically symmetric configuration $Y$ which is arbitrarily close to $X$ with the same $\varphi_{0}$ and $N$ but strictly smaller $m$.

In order to examine the stability of the equilibrium solution, we follow the turning point method 1720 . In the present problem, $m$ is a potential function, since the equilibrium solution is a stationary point of $m(\delta m=0)$ and the stable equilibrium solution is a minimal point of $m\left(\delta m=0\right.$ and $\left.\delta^{2} m>0\right)$. The asymptotic value of the scalar field $\varphi_{0}$ and baryon number $N$ form a two-dimensional control space. The equilibrium solutions are uniquely parametrized by two parameters, i.e., the central value of the scalar field, $\varphi_{c}$, and the central total baryonic density, $\tilde{\rho}_{c}$.

We adopt the following stability criteria [20]:

(i) The stability of $X\left(\varphi_{c}, \tilde{\rho}_{c}\right)$ can change typically only at a "turning point." Here the "turning point" ( $\left.\varphi_{c}^{0}, \tilde{\rho}_{c}^{0}\right)$ is a point where there exists a nontrivial vector $\left(\delta \varphi_{c}, \delta \tilde{\rho}_{c}\right)$ such that

$$
\begin{aligned}
\delta \varphi_{0} & =\left(\frac{\partial \varphi_{0}}{\partial \varphi_{c}}\right)_{\tilde{\rho}_{c}} \delta \varphi_{c}+\left(\frac{\partial \varphi_{0}}{\partial \tilde{\rho}_{c}}\right)_{\varphi_{c}} \delta \tilde{\rho}_{c}=0, \\
\delta N & =\left(\frac{\partial N}{\partial \varphi_{c}}\right)_{\tilde{\rho}_{c}} \delta \varphi_{c}+\left(\frac{\partial N}{\partial \tilde{\rho}_{c}}\right)_{\varphi_{c}} \delta \tilde{\rho}_{c}=0 .
\end{aligned}
$$

From Eqs. (3.7) and (3.8),

$$
\frac{\partial\left(\varphi_{0}, N\right)}{\partial\left(\varphi_{c}, \tilde{\rho}_{c}\right)}=0
$$

at the turning point. Therefore the change of stability can be detected as envelopes of a family of curves $\tilde{\rho}_{c}=$ const in the $\left(\varphi_{0}, N\right)$ plane. Of course, this is also true for a family of curves $\varphi_{c}=$ const.

(ii) In order to specify an unstable branch at the turning point, we draw the sequence of equilibrium solutions in the $\left(\varphi_{0}, \omega\right)$ plane, maintaining $N$ constant. Then, as one proceeds along the curve in a counterclockwise direction, a branch beyond the turning point is unstable. This is also the case with the curve $\left(N,-\mu_{e f f}\right)$ with $\varphi_{0}$ constant. This is a direct consequence of theorem I of 20 .

Here we describe the meaning of criterion (i) in the context of catastrophe theory. We regard the ADM energy $m$ as a function of three variables $\varphi_{0}, N$, and $\omega$. We take $\omega$ as a state variable. We consider an equilibrium space

$$
M_{m}=\left\{\left(\varphi_{0}, N, \omega\right) \mid\left(\frac{\partial m}{\partial \omega}\right)_{\varphi_{0}, N}=0\right\}
$$

and a control space

$$
\mathbf{R}^{2}=\left\{\left(\varphi_{0}, N\right)\right\}
$$

We define a catastrophe map

$$
\begin{aligned}
\chi_{m}: M_{m} & \longrightarrow \mathbf{R}^{2}, \\
\left(\varphi_{0}, N, \omega\right) & \longmapsto\left(\varphi_{0}, N\right) .
\end{aligned}
$$

A point $P \in M_{m}$ is called a singular point of $\chi_{m}$ if the Jacobian of $\chi_{m}$ vanishes at $P$. A point $Q \in \mathbf{R}^{2}$ is called a singular value if there is at least one singular point in $\chi_{m}^{-1}(Q)$. A bifurcation set $B_{m} \subset \mathbf{R}^{2}$ is a set of singular values. At a singular point $P \in M_{m}$, a vector normal the tangent space of $M_{m}$, which is 


$$
\left(\left(\frac{\partial^{2} m}{\partial \varphi_{0} \partial \omega}\right)_{N, \omega},\left(\frac{\partial^{2} m}{\partial N \partial \omega}\right)_{\omega, \varphi_{0}},\left(\frac{\partial^{2} m}{\partial \omega^{2}}\right)_{\varphi_{0}, N}\right)
$$

is parallel to the $\varphi_{0} N$ plane. Therefore, the set of singular points, $\Sigma_{m} \subset M_{m}$, satisfies

$$
\Sigma_{m}=\left\{\left(\varphi_{0}, N, \omega\right) \mid\left(\frac{\partial m}{\partial \omega}\right)_{\varphi_{0}, N}=\left(\frac{\partial^{2} m}{\partial \omega^{2}}\right)_{\varphi_{0}, N}=0\right\},
$$

and the bifurcation set $B_{m} \subset \mathbf{R}^{2}$ satisfies

$$
B_{m}=\left\{\left(\varphi_{0}, N\right) \mid\left(\frac{\partial m}{\partial \omega}\right)_{\varphi_{0}, N}=\left(\frac{\partial^{2} m}{\partial \omega^{2}}\right)_{\varphi_{0}, N}=0\right\} .
$$

The envelopes of the family of the curves $\tilde{\rho}_{c}=$ const in the $\left(\varphi_{0}, N\right)$ plane form a bifurcation set $B_{m}$ of the catastrophe map $\chi_{m}$ because the Jacobian of $\chi_{m}$ vanishes at points on the envelopes. Criterion (i) says that a sequence of the equilibrium solutions can change its stability only at the points of the bifurcation set.

From criteria (i) and (ii), we examine the stability of the equilibrium solutions of neutron stars in scalar-tensor theory. From the turning point method alone, however, we cannot say that an equilibrium solution is stable. Therefore the stability of an equilibrium solution must be investigated by perturbation study once for all. For this purpose, we examine the case in which $\alpha\left(\varphi_{0}\right)=0$ and $m / r_{s}$ is sufficiently small ( $\tilde{\rho}_{c}$ is sufficiently small). In this case, there is an equilibrium solution that is identical to that in general relativity. For this solution, the second-order variation of $m$ by regular, adiabatic, time-symmetric, and spherically symmetric perturbations with $\varphi_{0}$ and $N$ constant is

$$
\delta^{2} m=\text { general relativistic terms }+\frac{1}{2} \int_{0}^{\infty} d r e^{-\nu / 2}\left(1-\frac{2 \mu}{r}\right)^{-1 / 2} \zeta\left[-\frac{d^{2}}{d r_{*}^{2}}+V(r)\right] \zeta,
$$

where

$$
\begin{aligned}
\zeta & \equiv r \delta \varphi, \\
d r_{*} & \equiv e^{\nu / 2}\left(1-\frac{2 \mu}{r}\right)^{1 / 2} d r, \\
V(r) & \equiv \frac{1}{r}\left(1-\frac{2 \mu}{r}\right)\left[\frac{\nu^{\prime}}{2}-\frac{\mu^{\prime} r-\mu}{r(r-2 \mu)}\right] e^{\nu}-4 \pi \beta_{0}(-\tilde{\rho}+3 \tilde{p}) e^{\nu},
\end{aligned}
$$

and see Appendix B of [27 for the general relativistic terms. The general relativistic part is positive definite if $\Gamma>\Gamma_{c}$, and $\Gamma_{c} \rightarrow 4 / 3$ in the Newtonian limit [28]. The second term is positive definite because the eigenvalues of the operator, $-d^{2} / d r_{*}^{2}+V$, are all positive for an arbitrary coupling function $A(\varphi)$ if $m / r_{s}$ is sufficiently small, which has been shown in [23]. Therefore, the general relativistic equilibrium solution in which the central density is sufficiently small is stable for the case $\alpha\left(\varphi_{0}\right)=0$ if $\Gamma>\Gamma_{c} \simeq 4 / 3$.

\section{RESULTS}

Hereafter we restrict our attention to the coupling function of the quadratic form

$$
A(\varphi)=\exp \left(\frac{1}{2} \beta \varphi^{2}\right) \text {. }
$$

For this model, the solar-system experiments constrain the present cosmological value of the scalar field through Eqs. (2.14) and (2.15) as

$$
\left|\varphi_{0}\right| \lesssim 0.032|\beta|^{-1}
$$

and

$$
\left|\varphi_{0}\right| \lesssim \begin{cases}0.012(1+\beta)^{-1 / 2}|\beta|^{-1} & \text { for } \beta>-1, \\ 0.029|1+\beta|^{-1 / 2}|\beta|^{-1} & \text { for } \beta<-1,\end{cases}
$$

respectively. In particular, if $\varphi_{0}=0$, the post-Newtonian limit of this theory agrees completely with that of general relativity because $\alpha\left(\varphi_{0}\right)=0$. 


\section{A. $\beta \gtrsim-4.35$ case}

We present here the results of the case $\beta=-4$, but the features are basically common to the case $\beta \gtrsim-4.35$. Figure 1 shows $\tilde{\rho}_{c}=$ const curves in the $\left(\varphi_{0}, N\right)$ plane, where the equilibrium solutions have been determined in the manner described in Sec. II. At a point on an envelope of the family of the curves seen in Fig. 1, the stability of the sequence of equilibrium solutions changes. Figure 2 shows the curves $\left(\varphi_{0}, \omega\right)$ with $N$ constant. In Fig. 2, the solid lines denote stable branches while the dotted lines denote unstable branches, where stability criteria (i) and (ii) are applied. Therefore, in region (A) in Fig. 1, only one stable equilibrium solution exists. For $\varphi_{0}=0$, this stable solution is identical to that of general relativity. In region (B) in Fig. 1, however, no stable solution exists. This is classified as the fold catastrophe in which the control space is two-dimensional. This catastrophe is elementary and structurally stable. Hence it is expected that this catastrophe structure is not changed by adding small higher-order terms to the exponent of the coupling function (4.1). The potential function $m$ is written locally around point $p\left(\varphi_{0 p}, N_{p}\right)$ on the envelope (see Fig. 1) as, for $\varphi_{0 p}>0$,

$$
m=\frac{A}{3}\left(\omega-\omega_{p}\right)^{3}+\left[B\left(\varphi_{0 p}-\varphi_{0}\right)+B^{\prime}\left(N-N_{p}\right)\right]\left(\omega-\omega_{p}\right)+m_{p}
$$

where $A, B$, and $B^{\prime}$ are some positive constants. Then the terms in the square brackets cancel out on the envelope, and are negative in region (A) and positive in region (B). For $\varphi_{0 p}<0$, replace $\omega-\omega_{p}$ and $\varphi_{0 p}-\varphi_{0}$ with $\omega_{p}-\omega$ and $\varphi_{0}-\varphi_{0 p}$, respectively. For simplicity, we describe the behavior of the scalar charge for the case $\varphi_{0 p}>0$. From Eq. (4.4), near point $p$, the scalar charge is given by the roots of the following quadratic equation:

$$
\left(\frac{\partial m}{\partial \omega}\right)_{\varphi_{0}, N}=A\left(\omega-\omega_{p}\right)^{2}+\left[B\left(\varphi_{0 p}-\varphi_{0}\right)+B^{\prime}\left(N-N_{p}\right)\right]=0
$$

The scalar charge is then given near point $p$ in region $(\mathrm{A})$ by

$$
\omega=\omega_{p} \pm A^{-1 / 2}\left[B\left(\varphi_{0}-\varphi_{0 p}\right)+B^{\prime}\left(N_{p}-N\right)\right]^{1 / 2}
$$

where the upper sign denotes the stable branch and the lower denotes the unstable one. If a quadratic term in $\left(\omega-\omega_{p}\right)$ was involved in Eq. (4.4), the number of the roots of the equation $\partial m / \partial \omega=0$ did not change at point $p$. That is why Eq. (4.4) does not contain the quadratic term. The "scalar susceptibility" $\chi_{\varphi}$ is given near point $p$ by

$$
\chi_{\varphi} \equiv\left(\frac{\partial \omega}{\partial \varphi_{0}}\right)_{N}= \pm \frac{1}{2} A^{-1 / 2} B\left[B\left(\varphi_{0}-\varphi_{0 p}\right)+B^{\prime}\left(N_{p}-N\right)\right]^{-1 / 2} .
$$

The bifurcation set $B_{m} \subset \mathbf{R}^{2}$, which is the envelope seen in Fig. 1, is given by

$$
\begin{aligned}
& \left(\frac{\partial m}{\partial \omega}\right)_{\varphi_{0}, N}=A\left(\omega-\omega_{p}\right)^{2}+\left[B\left(\varphi_{0 p}-\varphi_{0}\right)+B^{\prime}\left(N-N_{p}\right)\right]=0, \\
& \left(\frac{\partial^{2} m}{\partial \omega^{2}}\right)_{\varphi_{0}, N}=2 A\left(\omega-\omega_{p}\right)=0
\end{aligned}
$$

i.e.,

$$
\varphi_{0}=\frac{B^{\prime}}{B}\left(N-N_{p}\right)+\varphi_{0 p}
$$

near point $p$. This fold catastrophe appears also in general relativity in which $A(\varphi)=1$ identically. In general relativity, because of the absence of a gravitational scalar field, the control space is one-dimensional. For $\beta=-4$, the maximum ADM energy is greater than the general relativistic one for $\varphi_{0} \neq 0$. This is because, due to the presence of the scalar field, the effective gravitational constant becomes smaller and thereby gravity becomes weaker than in general relativity.

\section{B. $\beta \lesssim-4.35$ case}

This case is more interesting than the above case. We present the results of the case $\beta=-6$. Figure 3 shows $\tilde{\rho}_{c}=$ const curves in the $\left(\varphi_{0}, N\right)$ plane. This figure is very different from Fig. 1 . On the envelope of the family of 
curves, $e^{\prime} d c b a b^{\prime} c^{\prime} d e$, the sequence of equilibrium solutions changes its stability. Although there are other envelopes in region (B), they have nothing to do with the change of the number of stable equilibrium solutions. Figure 4 shows curves $\left(\varphi_{0}, \omega\right)$ with $N$ constant. From criteria (i) and (ii), the number of stable equilibrium solutions is as follows: In region (A), only one stable equilibrium solution exists. In region (B), two distinct stable equilibrium solutions exist. Surprisingly, these stable equilibrium solutions are different even for $\varphi_{0}=0$ from their counterparts in general relativity. For $\varphi_{0}=0$, the unstable solution agrees with the stable solution in general relativity. One of the two stable equilibrium solutions disappears on the envelope $d c b a b^{\prime} c^{\prime} d$. In region $(\mathrm{C})$, no stable equilibrium solution exists. Point $a$ is a bifurcation point for $\varphi_{0}=0$. This is seen in Fig. 5 which displays the curves $\left(\tilde{\rho}_{c}, m\right)$ and $\left(\tilde{\rho}_{c}, N\right)$ for $\varphi_{0}=0$, where the solid lines denote stable branches and the dotted lines denote unstable branches. In this figure, two stable branches are degenerate because, for $\varphi_{0}=0$, two stable equilibrium solutions are identical except for the sign of the scalar field. The equilibrium solution of the bifurcated stable branches is more compact for smaller mass but less compact for larger mass than the general relativistic sequence. Figure 6 shows the equilibrium space $M_{m}$ near point $a$. This type of the catastrophe at point $a$ is classified as the cusp catastrophe in which the control space is two-dimensional. The map $\chi_{m}$ is a cusp catastrophe map. This catastrophe is elementary and structurally stable, which suggests that this structure is stable against adding small higher order terms to the exponent of the coupling function (4.1). Point $a$ is called a cusp point.

We restrict our attention to cusp point $a\left(0, N_{a}\right)$. $\left(m_{b} N_{a} \simeq 1.24 M_{\odot}\right.$ for $\beta=-6$.) The potential function $m$ is written around cusp point $a$ as

$$
m=\frac{C}{4} \omega^{4}-\frac{D\left(N-N_{a}\right)}{2} \omega^{2}-\varphi_{0} \omega+m_{a}
$$

where $C$ and $D$ are some positive constants. The reason why the coefficient of $\varphi_{0} \omega$ is determined is that Eq. (3.1) holds. This form of expansion agrees with the usual Landau ansatz for a second-order phase transition, which has been used to explain spontaneous scalarization by Damour and Esposito-Farèse [16]. The scalar charge is given by the roots of the following cubic equation:

$$
\left(\frac{\partial m}{\partial \omega}\right)_{\varphi_{0}, N}=C \omega^{3}-D\left(N-N_{a}\right) \omega-\varphi_{0}=0
$$

From Eq. (4.12), near cusp point $a$, the scalar charge is given by

$$
\omega=0
$$

for $N<N_{a}$ with $\varphi_{0}=0$. This is a stable branch. For $N>N_{a}$ with $\varphi_{0}=0$,

$$
\omega= \begin{cases} \pm\left(\frac{D}{C}\right)^{1 / 2}\left(N-N_{a}\right)^{1 / 2} & \text { for the stable branches, } \\ 0 & \text { for the unstable branch. }\end{cases}
$$

At point $a$ the stable equilibrium solution changes continuously, but its derivative with respect to $N$ is discontinuous. If Eq. (4.11) involved a cubic term in $\omega$, the number of roots of the equation $\partial m / \partial \omega=0$ did change at point $a$. But this catastrophe was classified as the fold type and therefore not the case for point $a$ because of the shape of the bifurcation set seen in Fig. 3. That is why Eq. (4.11) does not contain the cubic term. We also note that, for the case of two-dimensional control space, the structurally stable catastrophe is classified as either the fold or cusp type by Thom's theorem. Therefore, at point $a$, a second-order phase transition occurs. If we fix $N$ to $N_{a}$,

$$
\omega=C^{-1 / 3} \varphi_{0}^{1 / 3} .
$$

This is stable. From Eqs. (4.12)-(4.14), with $\varphi_{0}=0$ near point $a$, it is derived that the scalar susceptibility $\chi_{\varphi}$ is given by

$$
\chi_{\varphi}= \begin{cases}D^{-1}\left(N_{a}-N\right)^{-1} & \text { for } N<N_{a} \\ \frac{1}{2} D^{-1}\left(N-N_{a}\right)^{-1} & \text { for } N>N_{a}\end{cases}
$$

Near point $a$ in region (A) in Fig. 3, only one real root of the cubic equation (4.12) corresponds to the stable equilibrium solution, while, in region (B), the smallest and largest roots of three real roots correspond to the stable equilibrium solutions and the intermediate root corresponds to the unstable one. If $\varphi_{0}>0$, the largest root corresponds to the globally stable one. If $\varphi_{0}<0$, the smallest root corresponds to the globally stable one. If $\varphi_{0}=0$, the two stable equilibrium solutions have identical ADM energies. The bifurcation set $B_{m}$, which is the envelope $b^{\prime} a b$, is given by 


$$
\begin{aligned}
\left(\frac{\partial m}{\partial \omega}\right)_{\varphi_{0}, N} & =C \omega^{3}-D\left(N-N_{a}\right) \omega-\varphi_{0}=0, \\
\left(\frac{\partial^{2} m}{\partial \omega^{2}}\right)_{\varphi_{0}, N} & =3 C \omega^{2}-D\left(N-N_{a}\right)=0
\end{aligned}
$$

i.e.,

$$
\varphi_{0}= \pm\left(\frac{4 D^{3}}{27 C}\right)^{1 / 2}\left(N-N_{a}\right)^{3 / 2}
$$

near point $a$. The cusp catastrophe has been named after this shape. On the envelope $d c b a b^{\prime} c^{\prime} d$ except for points $a$ and $d$, one of the two distinct stable equilibrium solutions, the locally but not globally stable one, disappears, and hence a first-order phase transition occurs if the system obeys a perfect delay convention. On envelope $e d e^{\prime}$, the stable equilibrium solution disappears. The catastrophic feature on the envelopes except for point $a$ is the fold catastrophe described in the last subsection. Point $d$ is not a cusp point but the intersection of two folds.

We should comment that, for the near critical case $-4.9 \lesssim \beta \lesssim-4.35$, the behavior of the stable equilibrium solutions around the point of the maximum baryon number is somewhat complicated, although the structure of the cusp catastrophe at cusp point $a$ is not changed. Figures 7 and 8 show the curves $\left(\tilde{\rho}_{c}, N\right)$ with $\varphi_{0}=0$, for $\beta=-4.5$ and -4.85 , respectively. For $-4.8 \lesssim \beta \lesssim-4.35$, the number of stable equilibrium solutions changes as 1,2 (degenerate in Fig. 7), 3, 1, 0 as the control parameter $N$ is increased continuously, as is seen in Fig. 7. For $-4.9 \lesssim \beta \lesssim-4.8$, the number of stable equilibrium solutions changes as 1, 2 (degenerate in Fig. 8), 3, 2, 0 as $N$ is increased continuously, as is seen in Fig. 8. For $\beta \lesssim-4.6$ the maximum ADM energy with $\varphi_{0}=0$ is greater than that in general relativity, while, for $\beta \gtrsim-4.6$, it is the same as that in general relativity.

The Kepler mass, which governs the Newtonian orbital motion of a test body, is not the ADM energy $m$ in general, but [2]

$$
\tilde{\mu}=\frac{1+\alpha_{0} \alpha_{A}}{1+\alpha_{0}^{2}} m
$$

where

$$
\alpha_{A} \equiv \frac{\partial \ln m}{\partial \varphi}=-\frac{\omega}{m}
$$

When we consider the case of $\alpha_{0}=\beta \varphi_{0}=0$, the Kepler mass is identical to the ADM energy. Therefore the argument above for $\varphi_{0}=0$ is also valid for the Kepler mass.

Here we present the physical interpretation as to why spontaneous scalarization occurs. In spite of the absence of the potential in the Lagrangian, the scalar field $\varphi$ obtains an effective potential term $W(\varphi)$ which satisfies

$$
\frac{\partial W}{\partial \varphi}=-4 \pi \alpha(\varphi) T_{*}
$$

because of the coupling with matter. Note that $T_{*}$ depends on $\varphi$. Then, if we consider $A(\varphi)$ of the form (4.1),

$$
\frac{\partial V}{\partial \varphi}=-4 \pi \beta \varphi T_{*}
$$

and if $T_{*}=A^{4}(-\tilde{\rho}+3 \tilde{p})$ is negative, $\varphi=0$ is an unstable stationary point of the effective potential, if $\beta<0$. On the other hand, the term from the spatial derivative in Eq. (2.4) has a contribution to stabilize the solution. By these two competing effects, the stability of the trivial configuration $\varphi=0$ against spontaneous scalarization is governed. For a detailed analysis of the stability of the trivial configuration, see [23].

If spontaneous scalarization occurs, the effective gravitational constant, which is $A^{2}(\varphi)=\exp \left(\beta \varphi^{2}\right)$ in the sense of the inverse of the Brans-Dicke scalar field, becomes considerably smaller than unity. Thereby the gravitation becomes weaker and a considerably larger mass than in general relativity can be supported by the lower matter pressure than in general relativity. 


\section{SUMMARY AND DISCUSSIONS}

The behavior of the equilibrium solutions of neutron stars in scalar-tensor theories of gravitation shows a catastrophic feature, which is characterized by a discontinuous change of the system. When we consider a function $A(\varphi)$ of the form $A(\varphi)=\exp \left(\frac{1}{2} \beta \varphi^{2}\right)$, the catastrophe types are classified as fold and cusp catastrophes. The appearance of the cusp catastrophe depends on whether $\beta \gtrsim-4.35$ or $\beta \lesssim-4.35$. From the fact that those types of catastrophes are structurally stable, it is expected that they would be seen in a wide class of coupling functions.

For $\beta \gtrsim-4.35$, the fold catastrophe on the two-dimensional control space does occur. The critical baryon number and critical ADM energy depend on $\varphi_{0}$. For a baryon number smaller than the critical one, one stable equilibrium solution exists, while, for a baryon number larger than the critical one, no stable equilibrium solution exists. In particular, for $\varphi_{0}=0$, the stable equilibrium solution is completely identical to that in general relativity. The behavior of the scalar charge and scalar susceptibility near the critical baryon number is explained by the form of the potential function of the fold catastrophe.

For $\beta \lesssim-4.35$, the cusp catastrophe does occur while the fold catastrophe also occurs. For $\beta \lesssim-4.9$, there is some critical value of the scalar field, $\varphi_{0}^{\text {crit }}>0$. If $\left|\varphi_{0}\right|>\varphi_{0}^{\text {crit }}$, there is only one critical number $N^{\text {crit } 1}$ that depends on $\varphi_{0}$. For $N<N^{c r i t 1}$, one stable equilibrium solution exists, while, for $N>N^{c r i t 1}$, no stable equilibrium solution exists. If $0<\left|\varphi_{0}\right|<\varphi_{0}^{\text {crit }}$, there are three critical baryon numbers $N^{\text {crit } 1}>N^{\text {crit } 2}>N^{\text {crit } 3}$. For $N^{\text {crit }}<N^{\text {crit }}$ or $N^{\text {crit } 2}<N<N^{\text {crit } 1}$, only one stable equilibrium solution exists. For $N^{\text {crit } 3}<N<N^{\text {crit } 2}$, two distinct stable equilibrium solutions exist and they do not agree with those in general relativity even for the limit $\varphi_{0} \rightarrow 0$. The almost general relativistic branch is unstable for $N>N^{\text {crit2 }}$. For $N>N^{\text {crit } 1}$, however, no stable equilibrium solution exists. If $\varphi_{0}=0$, the sequence of equilibrium solutions of neutron stars bifurcates at a point. Beyond this point, the general relativistic branch becomes unstable and another two (degenerate) sequences of equilibrium solutions far from the general relativistic one are stable. This bifurcation point is a cusp point, and the behavior of the scalar charge and scalar susceptibility near the cusp point is explained by the form of the potential function of the cusp catastrophe. At a point on the envelopes other than the cusp point, the fold catastrophe occurs. Since the critical baryon numbers $N^{c r i t} 1$ and $N^{\text {crit2 }}$ agree, the number of stable equilibrium solutions is 1 for $N<N^{\text {crit } 3}, 2$ for $N^{\text {crit } 3}<N<N^{\text {crit } 2}=N^{\text {crit } 1}$ and 0 for $N^{\text {crit } 2}=N^{\text {crit } 1}<N$. It should be noticed that, for the near critical case $-4.9 \lesssim \beta \lesssim-4.35$, the structure of the cusp catastrophe does appear although the behavior becomes somewhat more complicated around the maximum baryon number for $\varphi_{0} \simeq 0$. This complicated feature agrees with the fact that the critical mass against zero-mode instability is not a monotonic function with respect to $\beta$, which is seen in Table I of [23].

Here we comment on the continuous change of the asymptotic value of the scalar field $\varphi_{0}$. If we identify $\varphi_{0}$ with the cosmological value of the scalar field, the evolution of $\varphi_{0}$ can be described by the equation of motion (2.4) in the Friedmann-Robertson-Walker universe. On the other hand, if we identify $\varphi_{0}$ with the value of the scalar field at the matching region in the $N$-compact-body problem, $\varphi_{0}$ should evolve due to the change of the density distribution around the neutron star. If the time scale of the variation of $\varphi_{0}$ is sufficiently longer than that of the local gravitational phenomena, such as the scalar gravitational wave radiation, the process due to the change of $\varphi_{0}$ can be regarded as quasistatic. Through the cosmological evolution of the scalar field $\varphi_{0}$, the neutron stars may collapse and radiate a scalar gravitational wave.

We also comment on the continuous change of $N$, which may be a result of a mass accretion onto the neutron star. If the baryon number of the neutron star exceeds the maximum value, the neutron star collapses and scalar gravitational waves are radiated and this is a candidate for the source of the scalar gravitational waves [12 14]. In a theory like the one of Fig. 7, there is a stable general relativistic neutron star that has the same baryon number and ADM energy within numerical accuracy as the maximum-mass non-general-relativistic neutron star has. Then, the transition of the non-general-relativistic neutron star to the general relativistic one due to a mass accretion occurs without any energy extraction.

Scalar-tensor theories of gravity naturally arise from the low-energy limit of string theory or other unified theories. For the moment, however, it is not clear how the scalar fields should couple to gravity (but see [29]). Experimental tests, such as binary pulsar timing observations, may constrain the way of coupling between the scalar fields and gravity. In particular, as for the case in which the single, massless scalar field couples to gravity with the coupling function $A(\varphi)=\exp \left[(1 / 2) \beta \varphi^{2}\right]$, Damour and Esposito-Farèse [16] obtained the constraint on $\beta$ as $\beta \gtrsim-5$, using the data of three binary pulsars. They showed that the occurrence of spontaneous scalarization makes it very difficult for the theory to maintain consistency with the results of binary pulsar timing experiments. The results obtained in this paper show that spontaneous scalarization is not an exceptional but robust phenomenon for the neutron star and common to a wide range of coupling functions. Gravitational experiments with high-precision and/or in a strong-field regime and gravitational wave observations may have the potential to constrain the way of coupling of the gravitational scalar fields and thereby we may catch a glimpse of string-scale physics. 


\section{ACKNOWLEDGMENTS}

I would like to thank T. Nakamura, M. Sasaki, Y. Eriguchi, N. Sugiyama, K. Nakao, M. Siino, T. Chiba, and M. Kaneko for useful discussions. I am also grateful to H. Sato for his continuous encouragement.

[1] C. M. Will, Theory and Experiment in Gravitational Physics, revised ed. (Cambridge University Press, Cambridge, England, 1993).

[2] T. Damour and G. Esposito-Farèse, Class. Quantum Grav. 9, 2093 (1992).

[3] C. Brans and R.H. Dicke, Phys. Rev. 124, 925 (1961).

[4] M.B. Green, J.H. Schwarz, and E. Witten, Superstring Theory, (Cambridge University Press, Cambridge, England, 1987), Vols. 1 and 2.

[5] P.J. Steinhardt and F.S. Accetta, Phys. Rev. Lett. 64, 2740 (1990).

[6] A. Abramovici et al., Science, 256, 325 (1992).

[7] C. Bradaschia et al.. Nucl. Instrum. Methods Phys. Res. A 289, 518 (1990).

[8] J. Hough, in Proceedings of the Sixth Marcel Grossmann Meeting on General Relativity, Kyoto, Japan, 1991 , edited by H. Sato and T. Nakamura (World Scientific, Singapore, 1992), p. 192.

[9] K. Kuroda et al., in Proceedings of International Conference on Gravitational Waves: Sources and Detectors, Pisa, Italy, , 1996, edited by I. Ciufolini and F. Fidecaro (World Scientific, Singapore, 1997), p. 100.

[10] C.M. Will, and H.W. Zaglauer, Astrophys. J. 346, 366 (1989).

[11] C.M. Will, Phys. Rev. D 50, 6058 (1994).

[12] M. Shibata, K. Nakao, and T. Nakamura, Phys. Rev. D 50, 7304 (1994).

[13] M.A. Scheel, S.L. Shapiro, and S.A. Teukolsky, Phys. Rev. D 51, 4208 (1995); 51, 4236 (1995).

[14] T. Harada, T. Chiba, K. Nakao, and T. Nakamura, Phys. Rev. D 55, 2024 (1997).

[15] T. Damour and G. Esposito-Farèse, Phys. Rev. Lett. 70, 2220 (1993).

[16] T. Damour and G. Esposito-Farèse, Phys. Rev. D 54, 1474 (1996).

[17] J. Katz, Mon. Not. R. Astron. Soc., 183, 765 (1978).

[18] J. Katz, Mon. Not. R. Astron. Soc., 189, 817 (1979).

[19] R. Sorkin, Astrophys. J. 249, 254 (1981).

[20] R. Sorkin, Astrophys. J. 257, 847 (1982).

[21] G.L. Comer and H. Shin-kai, gr-qc/9708071, Class. Quantum Grav. (to be published).

[22] D.F. Torres, A.R. Liddle, and F.E. Schunk, gr-qc/9710048.

[23] T. Harada, Prog. Theor. Phys. 98, 359 (1997).

[24] C.W. Misner, K.S. Thorne, and J.A. Wheeler, Gravitation (Freeman, New York, 1973).

[25] D.E. Lebach et al., Phys. Rev. Lett. 75, 1439 (1995).

[26] J.G. Williams, X.X. Newhall, and J.O. Dickey, Phys. Rev. D 53, 6730 (1996).

[27] B.K. Harrison, K.S. Thorne, M. Wakano, and J.A. Wheeler, Gravitation Theory and Gravitational Collapse (The University of Chicago Press, Chicago, 1964).

[28] S. Chandrasekhar, Astrophys. J. 140, 417 (1964).

[29] T. Damour and A.M. Polyakov, Nucl. Phys. B423, 532 (1994); Gen. Relativ. Gravit. 26, 1171 (1994). 


\section{FIGURE CAPTION}

Fig.1. A family of curves of $\tilde{\rho}_{c}=$ const in the $\left(\varphi_{0}, N\right)$ plane for the $\beta=-4$ case. The ordinate is $m_{b} N$ in place of the baryon number $N$. In region (A), only one stable equilibrium solution exists, while, in region (B), no stable equilibrium solution exists. At a point on the envelope of the family of the curves, the fold catastrophe occurs.

Fig.2. Curves $\left(\varphi_{0}, \omega\right)$ with $N$ constant for the $\beta=-4$ case. The number attached to each curve is $m_{b} N$ in the solar mass unit. The solid lines denote stable branches and the dotted lines denote unstable branches.

Fig.3. Same as Fig. 1, but for $\beta=-6$ case. In region (A), only one stable equilibrium solution exists. In region (B), two distinct stable equilibrium solutions exist. In region (C), no stable solution exists. Point $a$ is a cusp point. At point $a$, the cusp catastrophe occurs, while the fold catastrophe occurs at a point on the envelopes except for $a$.

Fig.4. Same as Fig. 2, but for the $\beta=-6$ case.

Fig.5. (a) $\left(\tilde{\rho}_{c}, m\right)$ and (b) $\left(\tilde{\rho}_{c}, N\right)$ curves with $\varphi_{0}=0$ for $\beta=-6$. The solid lines denote stable branches, while the dotted lines denote unstable branches. The two distinct bifurcated branches are degenerate because they have identical ADM energies and baryon numbers but scalar fields of the opposite sign. The number of stable equilibrium solutions changes as $1,2,0$ as $N$ increases.

Fig.6. Equilibrium space $M_{m}$ in the $\left(\varphi_{0}, N, \omega\right)$ space around cusp point $a$ for the $\beta=-6$ case. This structure of the equilibrium space is classified as the cusp catastrophe.

Fig.7. Same as Fig. 5(b), but for $\beta=-4.5$. The number of stable equilibrium solutions changes as 1, 2, 3, 1,0 as $N$ increases. Two solutions are degenerate on the non-general-relativistic branches.

Fig.8. Same as Fig. 5(b), but for $\beta=-4.85$. The number of stable equilibrium solutions changes as $1,2,3,2,0$ as $N$ increases. Two solutions are degenerate on the non-general-relativistic branches. 


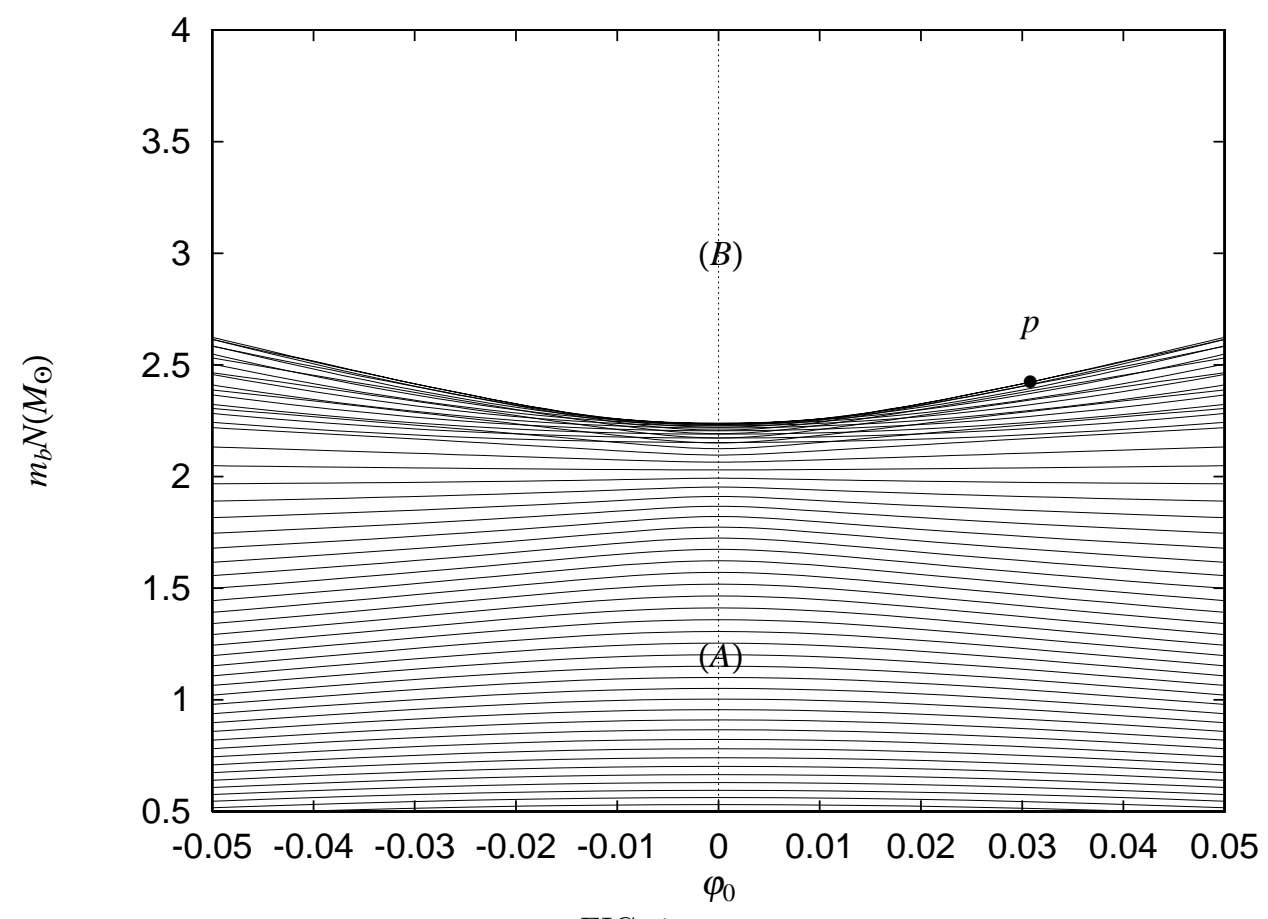

FIG. 1.

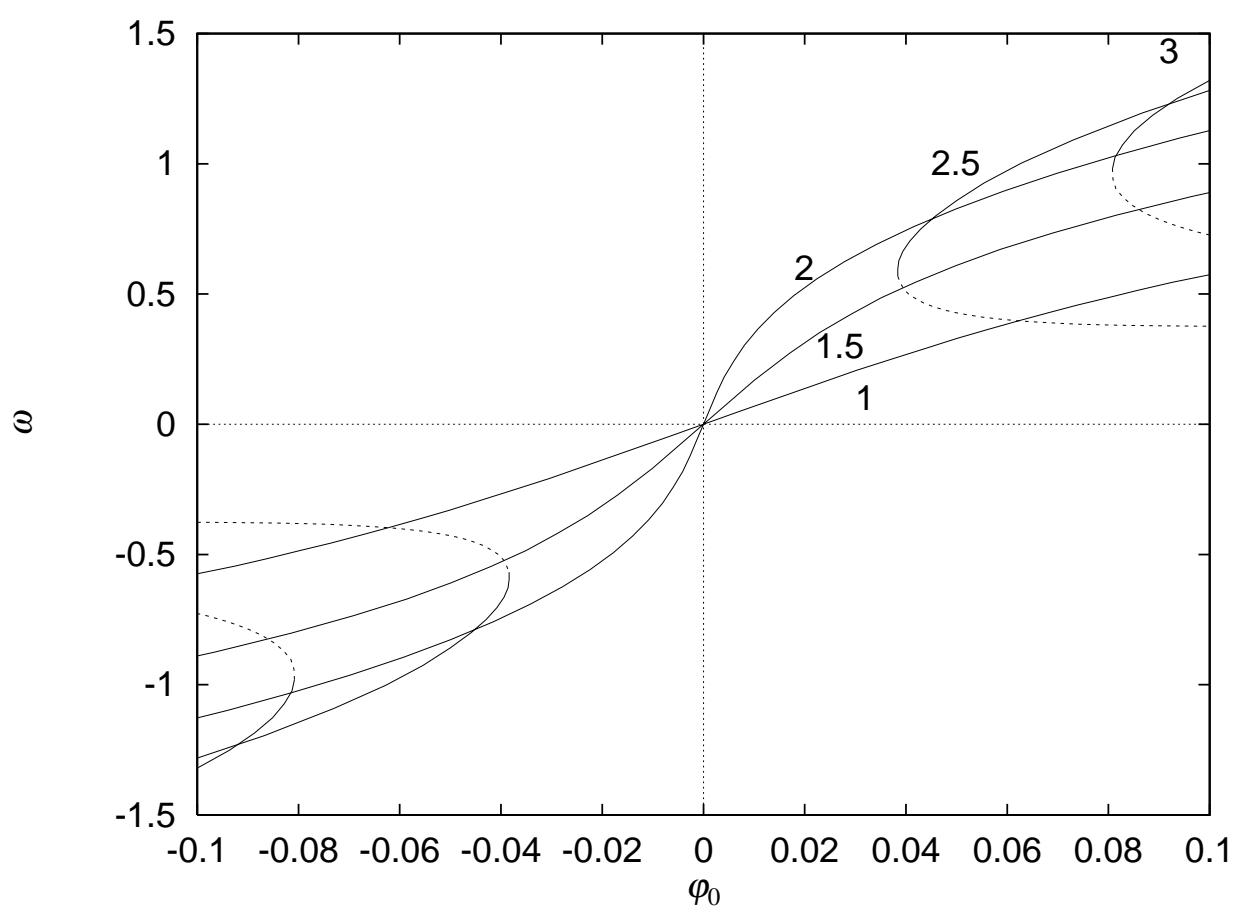

FIG. 2. 


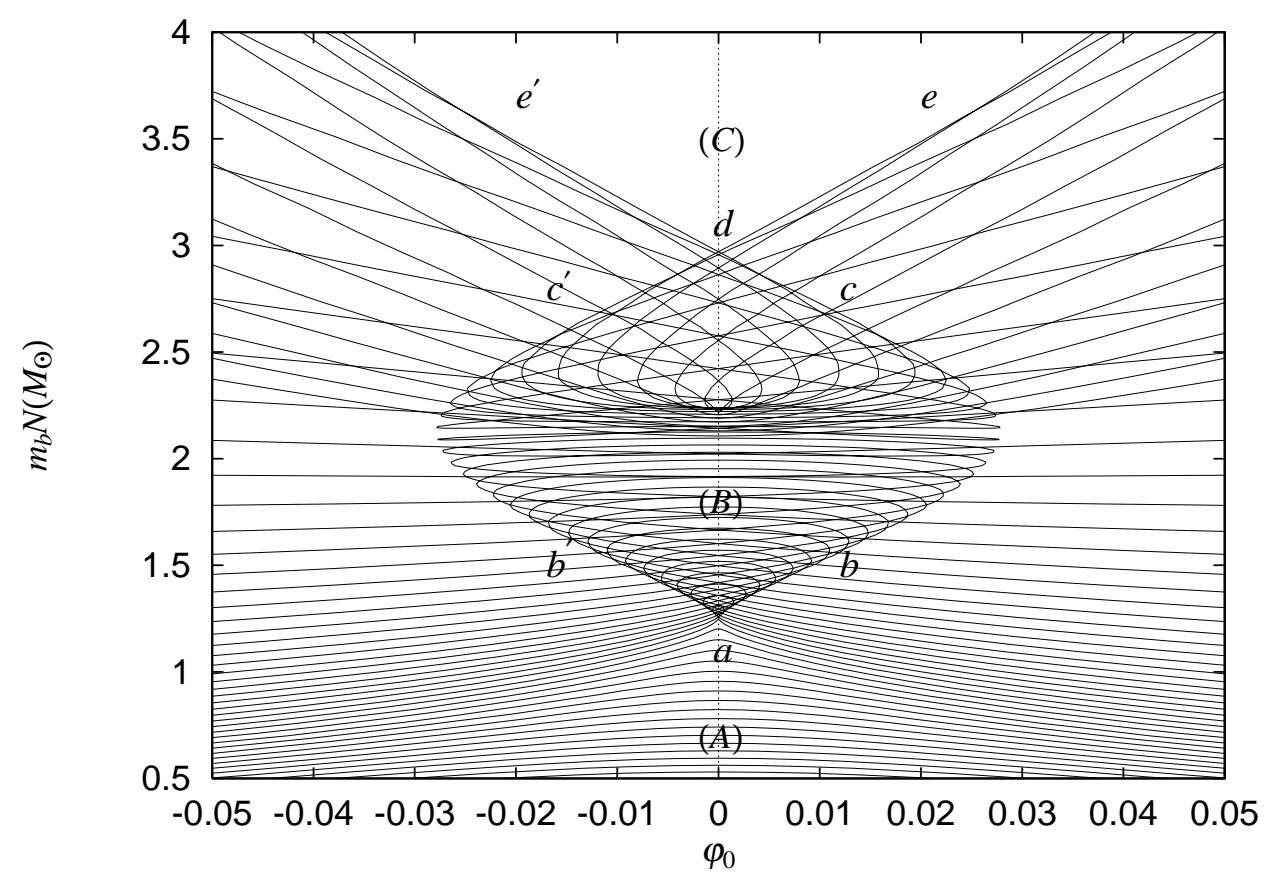

FIG. 3.

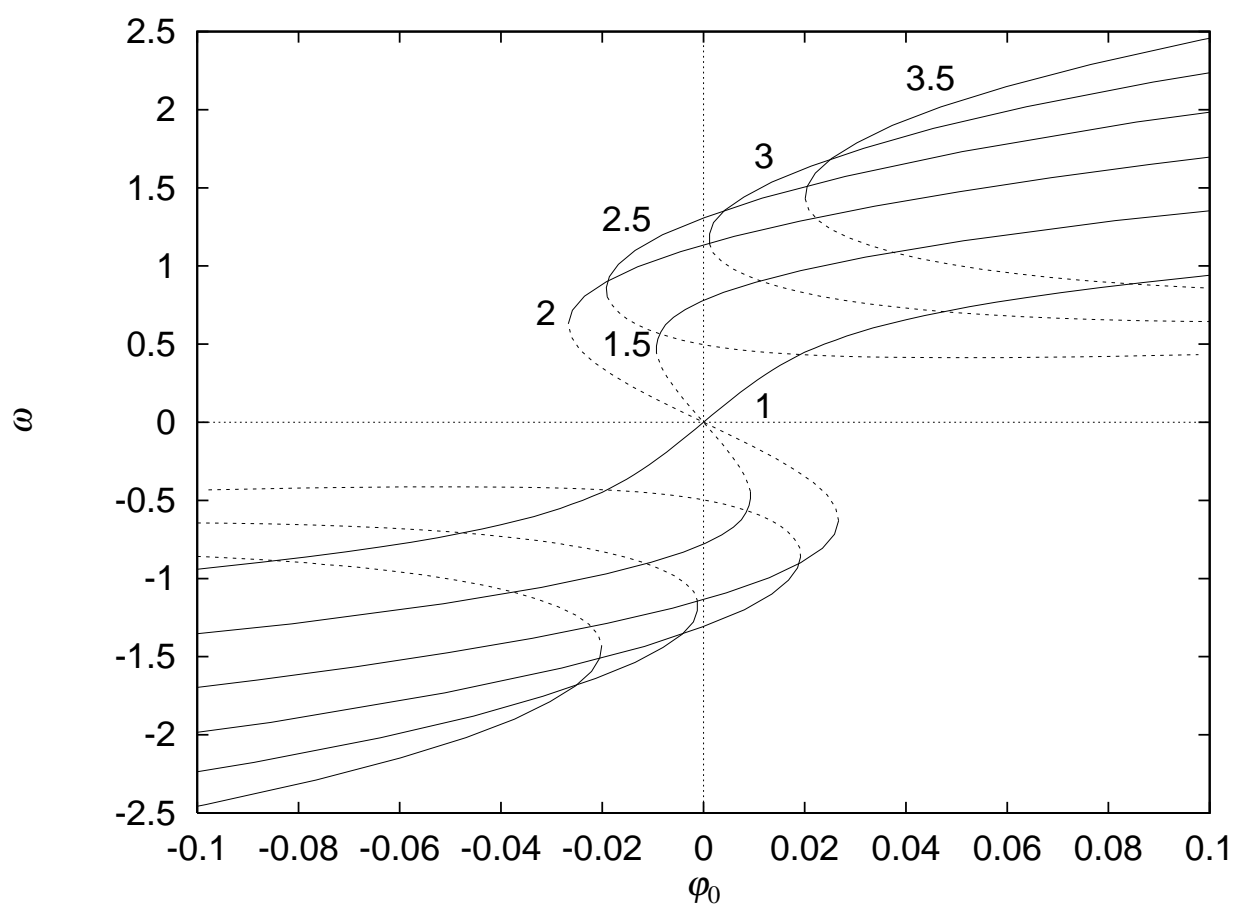

FIG. 4. 

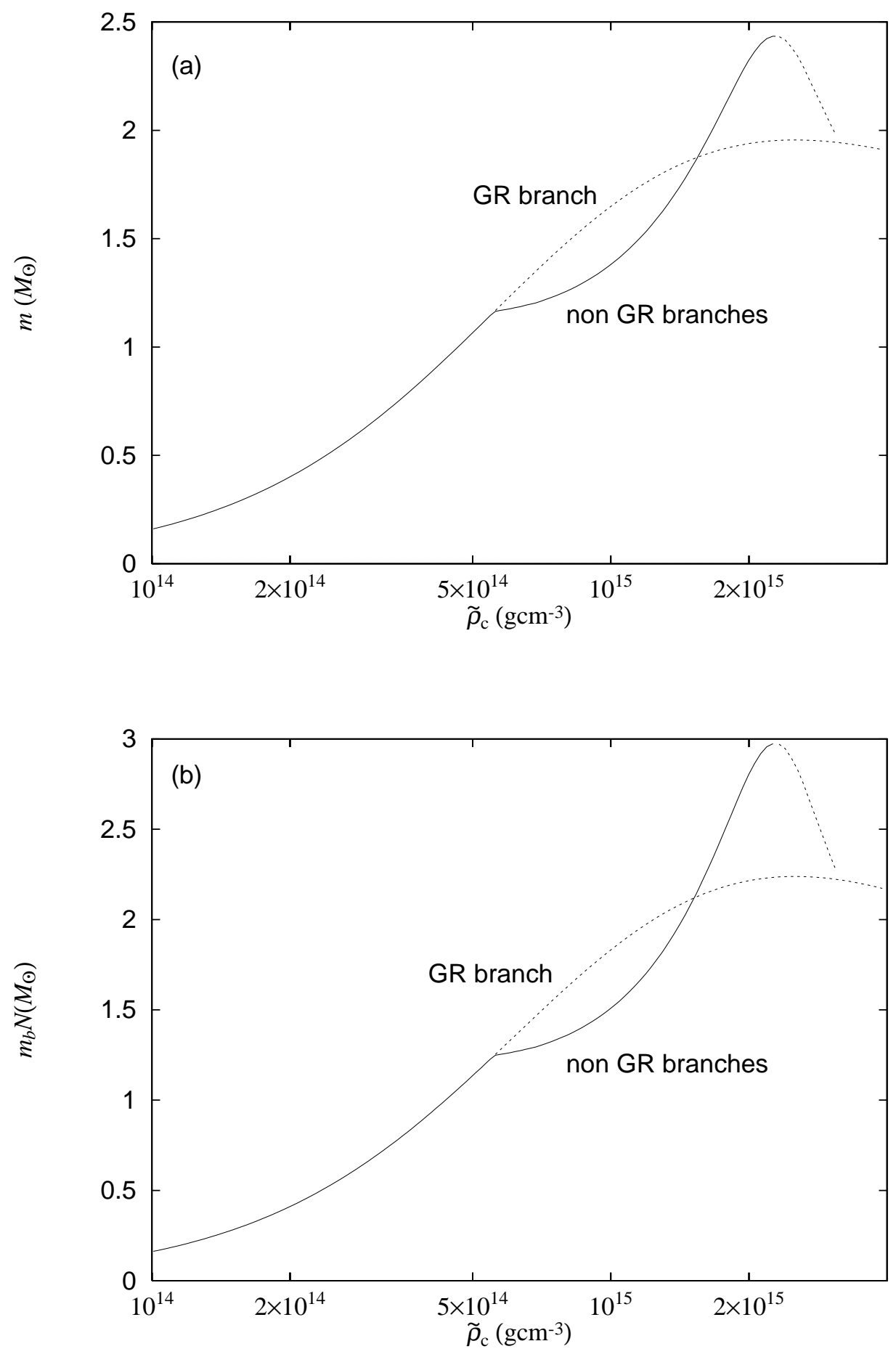

FIG. 5 . 


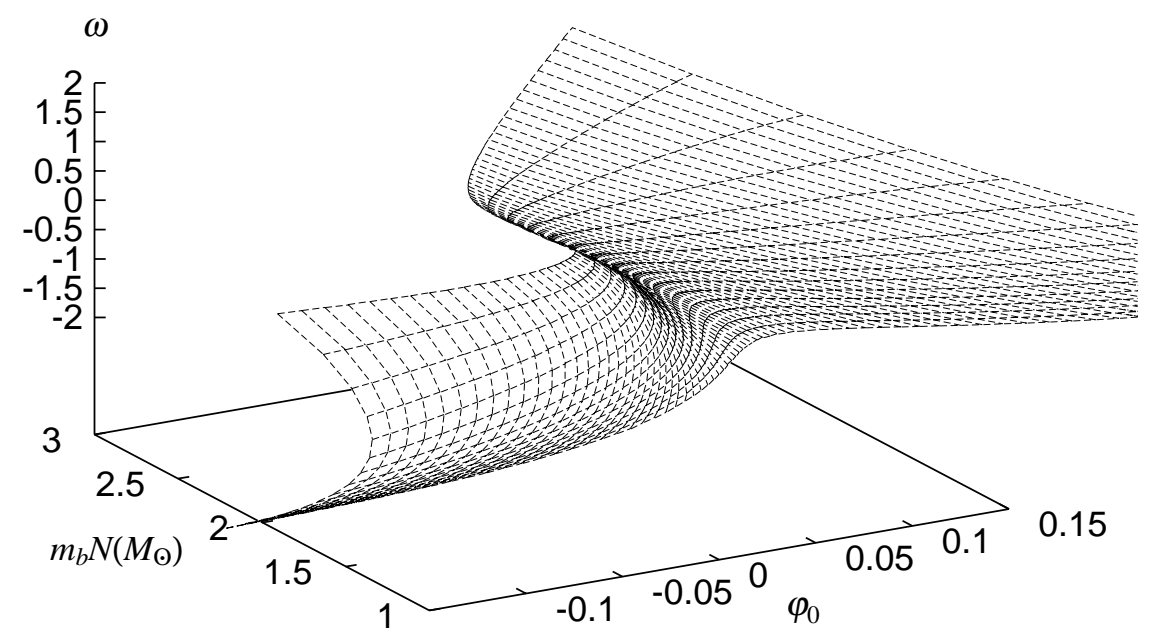

FIG. 6.

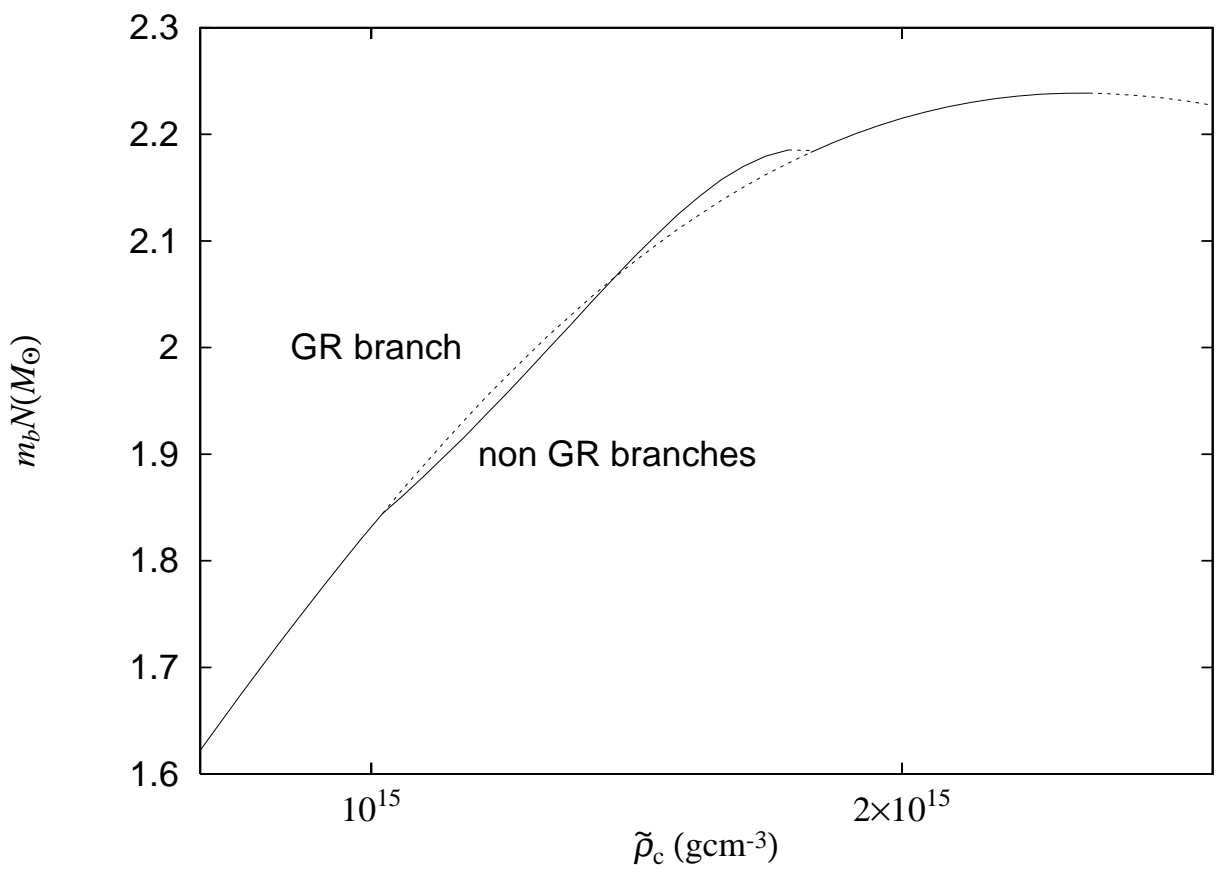

FIG. 7. 


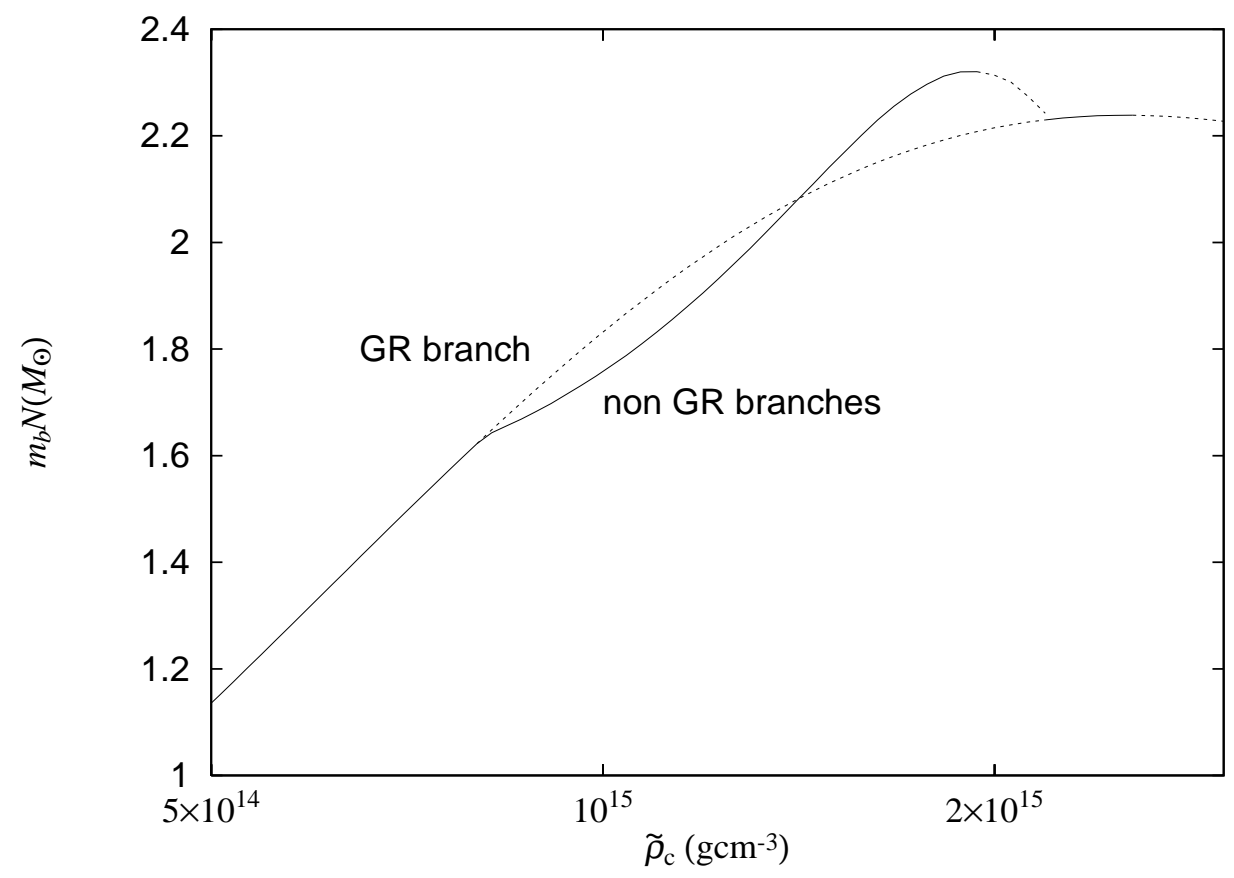

FIG. 8 